ANEXO No. 1

\title{
UNIDADES DE GESTIÓN ACADÉMICA COMO EJERCICIO DEMOCRÁTICO PARA EL LIDERAZGO Y LA GOBERNANZA INSTITUCIONAL EN IES MULTICAMPUS DE ALTA CALIDAD -Caso: Universidad Santo Tomás-
}

\section{Ponentes:}

Fray Eduardo González Gil, O.P., Vicerrector Académico General Fabiola Inés Hernández Barriga, Directora Unidad de Desarrollo Curricular y Formación Docente, Sede Principal Bogotá

Lina María Fonseca Ortiz, Docente Unidad de Desarrollo Curricular y Formación Docente, Sede Principal Bogotá

\section{Resumen ejecutivo de la Práctica:}

La Universidad Santo Tomás -USTA-, de carácter católica, privada, fundada en 1583, con presencia nacional en 5 ciudades y 23 municipios, más de 30000 estudiantes, 2500 docentes, 1600 administrativos, 145000 egresados, 200 programas académicos de pregrado y posgrados en las diferentes modalidades, promueve la formación integral como fin último de su Misión institucional, sustentada en el Proyecto Educativo Institucional -PEI-, el Modelo Educativo Pedagógico -MEPdonde la pedagogía con enfoque problémico y la metodología problematizadora, son rasgos identitarios de su acto educativo.

La USTA desde el año 2000 da sus primeros pasos en clave de gestión de lo que a 2016 sería una universidad con acreditación de alta calidad Multicampus. Es así como, en el marco de la gobernanza, entre el periodo 2012 y 2019 logra gestionar sus procesos académicos y de aseguramiento de la calidad, a través de la estructuración y consolidación de seis (6) Unidades Académicas adscritas a la Vicerrectoría Académica General, que apoyan, acompañan, articulan y asesoran el desarrollo de las funciones universitarias a nivel nacional: Unidad de Desarrollo Curricular y Formación Docente-UDCFD-; Unidad de Gestión Integral de la Calidad 
Universitaria -UGICU-; Unidad de Desarrollo Integral Estudiantil -UDIES-; Unidad de Investigación; Unidad de Proyección Social; Unidad de Posgrados. En línea con ello, se formularon políticas, lineamientos, procesos, procedimientos, entre otros, que garantizan el aseguramiento de la calidad en el marco de un Sistema Académico Integrado, a la luz de lo estipulado en la normatividad Ministerial e Institucional vigente.

Surgen tres niveles estructurales que marcan el derrotero para la constitución y funcionalidad de estas unidades de apoyo: superestructura, estructura e infraestructura, lo que posibilita diseñar un Modelo Institucional de Gestión Universitaria.

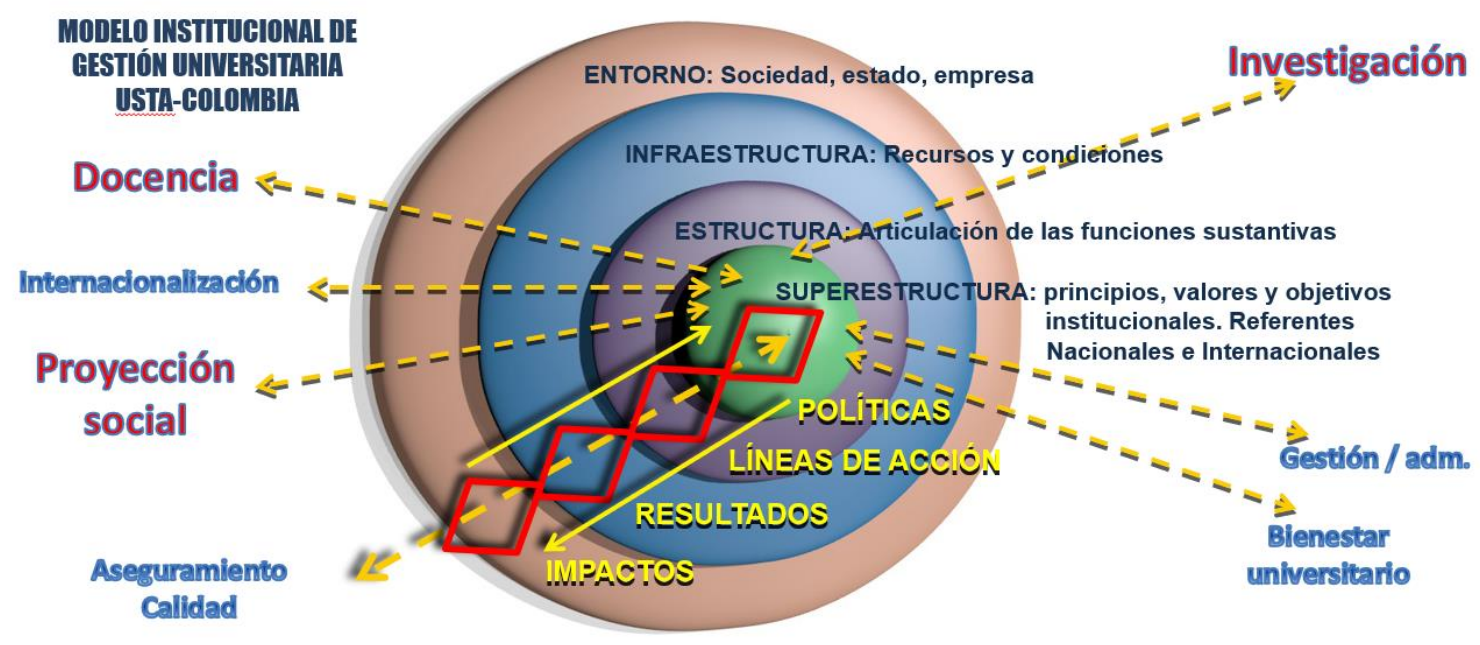

Modelo Institucional de Gestión Universitaria, USTA Colombia: 2015.

El Modelo Institucional se construye sobre tres ejes dimensionales: el nivel de superestructura, que se refiere a las intenciones de la universidad que se evidencian en los principios y valores de la filosofía institucional, y en los referentes y lineamientos nacionales e internacionales sobre los cuales se cimenta el devenir de la institución. El nivel de estructura, "hace referencia a las formas de organización del trabajo y las relaciones entre objetos, sujetos y circunstancias, en que las intenciones se hacen realidad en el proceso de enseñanza-aprendizaje y en las 
funciones sustantivas de la universidad" (González et ál., 2006), lo que implica la articulación entre las mismas y las funciones adjetivas. El nivel de infraestructura, son los recursos y condiciones que se requieren para el desarrollo de las funciones universitarias. A su vez, cada uno de estos niveles puede extrapolarse a la gestión de los programas académicos de las diferentes dependencias e instancias, y a los mismos espacios académicos o asignaturas, centros y grupos de investigación. El análisis de las tres dimensiones estructurales permite evaluar si la intencionalidad (superestructura) de cada función universitaria y sus respectivos procesos cuentan con el soporte organizativo y de estructura de trabajo (estructura), de recursos y condiciones (infraestructura) para responder a las exigencias que el entorno (Estado, sociedad y empresa) le plantea a la USTA. El resultado de este análisis posibilita que cada función universitaria diseñe políticas de gestión y líneas de acción que desarrollen las intencionalidades expresadas en dichas políticas, para proyectar y generar los resultados que se pretende impacten el entorno.

En sintonía con el Modelo, actualmente se cuenta con Documentos Marco que presentan el horizonte y las pautas para el desarrollo y unificación de procesos, estrategias, estructuras, que orientan el quehacer universitario, a los que subyace el PEI para el cumplimiento de la Misión de la USTA.

En este sentido, la Vicerrectoría Académica General -VAG, como líder y responsable de los procesos académicos, investigativos, de proyección social y de aseguramiento de la calidad, e inspirada en el Método Prudencial de Tomás de Aquino, (ver, juzgar, optar-actuar), orienta el desarrollo y articulación de las funciones universitarias a nivel Multicampus, a fin de garantizar la formación integral de los estudiantes, bajo el lema de su Plan Integral Multicampus -PIM (2016-2027) "comprometidos con la excelencia". Es así como, la USTA se constituye como Sistema Académico Integrado que reconoce la diversidad y multiculturalidad de las regiones donde hace presencia, sin perder la identidad institucional, en aras de promover la transformación social. 
En correspondencia, la USTA a nivel Multicampus cuenta con Políticas y Lineamientos de Investigación, innovación y creación artística y cultural; de Diseño y actualización curricular; de Acompañamiento y promoción de los estudiantes tomasinos; de Responsabilidad Social Universitaria; de Posgrados; de un Sistema de Aseguramiento de la Calidad, que coadyuvan con la gestión académicoadministrativa Institucional, con una perspectiva sistémica para la toma de decisiones encaminadas hacia un mismo fin. Estos logros se gestan en el seno de las unidades académicas, que desde un ejercicio democrático y de liderazgo, hacen parte estructural y funcional de la acción Multicampus.

\section{Planificación de la Práctica:}

En el 2000 a través del Consejo Superior se crean con carácter de órganos vicerrectorales y supradecanales cuatro (4) unidades académicas: Planeación Académica y Perfeccionamiento Docente; Investigación y Posgrados; Proyección Social y Extensión Universitaria; Evaluación y Regulación Académica. Luego de siete años y en el marco de la perfectibilidad de los procesos, estas se han consolidado, reestructurado y creado otras, a saber: en el 2007 se crea la Unidad de Gestión de la Calidad Universitaria, que en el 2011 ajusta su denominación y alcance: Unidad de Gestión Integral de la Calidad Universitaria -UGICU; para el mismo año, la Unidad de Planeación Académica y Perfeccionamiento Docente, se reestructura y ajusta su denominación: Unidad de Desarrollo Curricular y Formación Docente -UDCFD; de igual forma sucede con la Unidad de Investigación y Posgrados, que se separa creando dos unidades independientes. En 2012 se crea en modalidad experimental por un año, la Unidad de Desarrollo Integral Estudiantil -UDIES-, transcurrido el año, con base en el informe de pertinencia de la referida Unidad y observada la exitosa labor realizada, se aprobó de forma definitiva.

Así se ha consolidado la gestión académica como ejercicio democrático para el liderazgo y la gobernanza institucional, a través de la creación de Unidades Académicas. En coherencia se crea el Comité de Unidades Académicas Institucionales, con carácter asesor y cuyos propósitos se centran en planear, 
proyectar e integrar interdisciplinariamente los procesos de las Unidades Académicas y de los programas de la USTA en las áreas de su competencia.

En esta ruta de planeación académica, desde la Vicerrectoría Académica General, se parte de un diagnóstico que permite la identificación de una línea base del funcionamiento y gestión académico-administrativa de las Unidades Académicas, con lo cual desde el referido método prudencial, se posibilita ver críticamente la realidad universitaria, promover la gestión de las referidas Unidades, juzgar y contrastar la realidad con diversos referentes y criterios, tanto Ministeriales como Institucionales, a fin de tener en consideración las tendencias locales, nacionales e internacionales, para la planeación, gestión, seguimiento académico-administrativo de la USTA; para luego, optar desde la elección hecha a partir de la valoración de diferentes posibilidades de solución a problemáticas reales contextuales, con el objetivo de finalmente actuary hacer realidad la opción decantada, a través de rutas metodológicas de acompañamiento, asesoría, apoyo y articulación Multicampus.

Cada unidad planea su ejercicio de gestión académica con base en las políticas institucionales, y siempre a la luz de herramientas macro:

- Una planeación institucional de carácter multicampus: Plan Integral Multicampus 2016-2027

- Un Plan General de Desarrollo en cuatrienios: PGD 2016-2019

- Una planeación para cada sede o seccional, en correspondencia con el cuatrienio del PGD de la USTA: Plan de Desarrollo 2016-2019

- Un plan de acción trabajado por proyectos de desarrollo de cada unidad, con vigencias anuales.

\section{Desarrollo y ejecución de la Práctica:}

En el marco del desarrollo de la buena práctica, la Vicerrectoría Académica General vela por la articulación sistemática del aseguramiento de la calidad institucional en 
concordancia con lo establecido en el Plan Integral Multicampus (2016-2027) y en el Plan General de Desarrollo (2016- 2019). Es así como desde hace 19 años a través de las Unidades Académicas, la VAG apoya, articula, acompaña y asesora la gestión académico-administrativa de los facultades, programas académicos y departamentos transversales, a nivel Multicampus, en relación con el desarrollo de las funciones sustantivas y adjetivas; evidencia de ello hoy, se encuentra una USTA sólida que permanentemente se cualifica y propende por la formación integral de sus estudiantes.

El desarrollo de los proyectos académicos adscritos a las Unidades de gestión de la Vicerrectoría Académica General, se da desde la articulación de los procesos Institucionales en clave de la Política de Calidad Institucional, donde los procesos de direccionamiento, estratégicos, de apoyo y misionales, facultan el reconocimiento y reformulación de planes y acciones desde la Misión y la Visión de la USTA. 


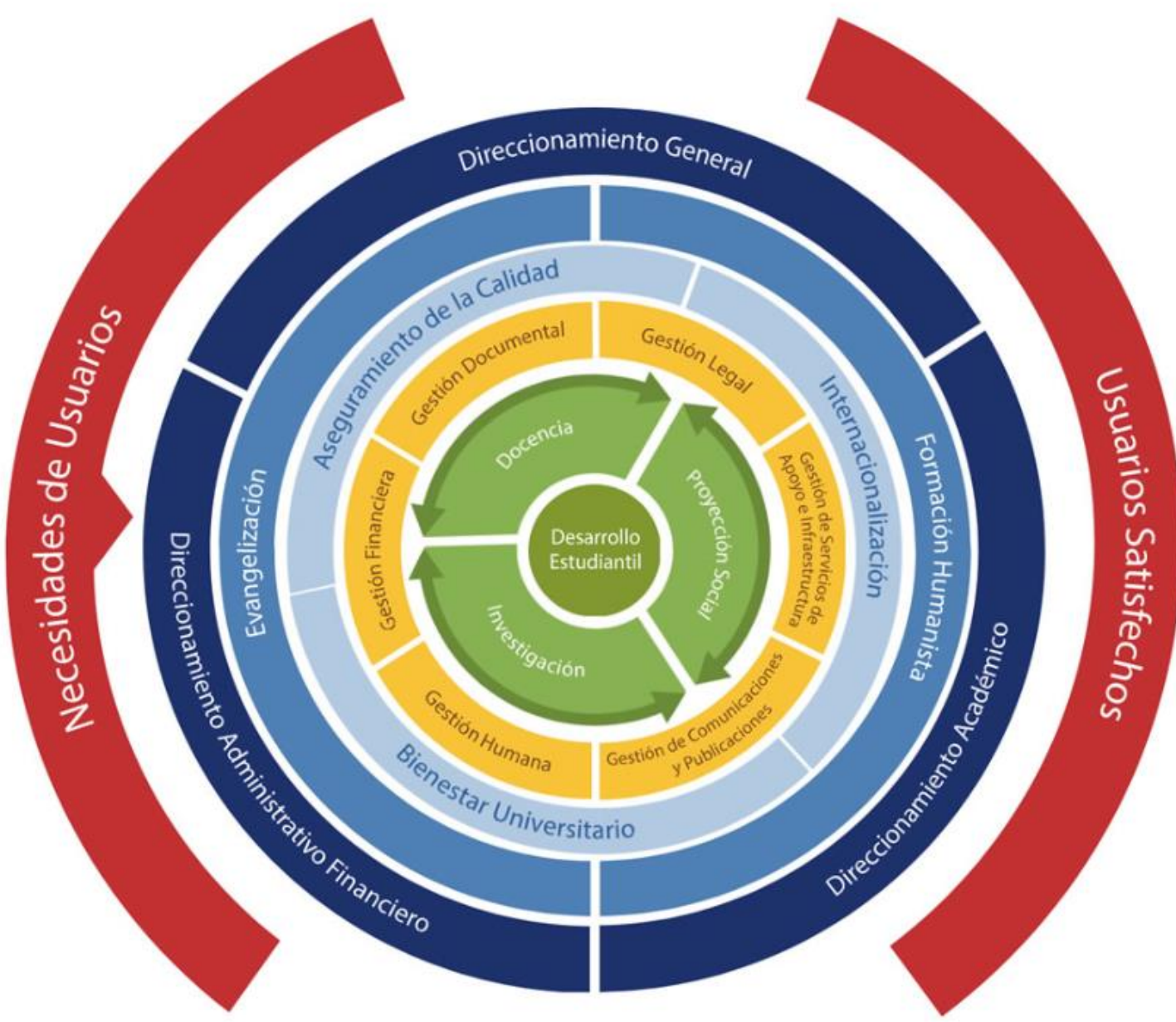

Mapa de procesos, USTA: 2015.

La Vicerrectoría Académica General y su staff de Unidades Académicas cuenta con un presupuesto anual para el desarrollo y gestión de sus proyectos, en este sentido, para las dos últimas vigencias fue de más de $\$ 9.000 .000 .000$, para la Sede Principal Bogotá, lo que ha permitido agenciar el desarrollo de planes, proyectos y acciones orientadas a la solución de problemáticas y necesidades institucionales Multicampus, y por ende a las del país. 


\begin{tabular}{|c|c|c|c|}
\hline UNIDAD & $\begin{array}{l}\text { Centro de } \\
\text { Costo }\end{array}$ & 2017 & 2018 \\
\hline Unidad de Investigación & 17100501 & 725.946 .767 & $\$ 1.430 .357 .345$ \\
\hline Unidad de Desarrollo Curricular y Formación Docente & 17150502 & $\$ 1.139 .155 .085$ & $\$ 1.276 .523 .056$ \\
\hline Unidad de Gestión Integral de la Calidad Universitaria & 17150501 & 749.339 .718 & 882.149 .975 \\
\hline Unidad de Proyección y Extensión Universitaria & 17200501 & 707.110.611 & 764.989 .402 \\
\hline Unidad de Desarrollo Integral Estudiantil & 17050508 & 621.237 .385 & 563.529 .314 \\
\hline Unidad de Posgrados & 17050509 & 438.092 .355 & 522.638 .014 \\
\hline TOTAL & & $\$ 4.380 .881 .921$ & $\$ \quad 5.440 .187 .105$ \\
\hline
\end{tabular}

Fuente: Departamento de Contabilidad y Presupuesto, 2019, Sede Principal Bogotá

Para el funcionamiento de las Unidades Académicas adscritas a la -VAG-, cada una cuenta con una estructura organizativa que le permite apoyar la gestión académicoadministrativa de las facultades y departamentos académicos. En este sentido se lideran desde una dirección que orienta, coordina y promueve el desarrollo de políticas institucionales relacionadas con las funciones universitarias. Cada dirección cuenta con un equipo de trabajo interdisciplinario con formación de base en Ciencias de la Educación, pues se entiende que el ejercicio que les subyace corresponde al reconocimiento de la educación superior, con el objetivo de velar por el aseguramiento de la calidad que les convoca, así como con personal de apoyo a la gestión propiamente administrativa y logística.

A continuación se presenta el organigrama de una de las Unidades Académicas adscritas a la VAG, a saber: Unidad de Desarrollo Curricular y Formación Docente -UDCFD-, cuya misión a nivel Multicampus es: Acompañar la gestión curricular de la USTA Colombia, a partir de la propuesta de políticas, procesos y procedimientos que concreten las intencionalidades declaradas en el Proyecto Educativo Institucional -PEI, para crear y consolidar programas académicos flexibles, integrales, interdisciplinarios e internacionalizados, que respondan con pertinencia y calidad, a las demandas de los contextos culturalmente diversos; así mismo, promueve el fortalecimiento y la consolidación del proyecto de vida del docente tomasino, como actor curricular, y favorece una carrera docente situada en su reconocimiento como persona y en su formación permanente. La gestión 
curricular y el desarrollo docente privilegian la formación integral del estudiante tomasino, con la que se compromete la Universidad.

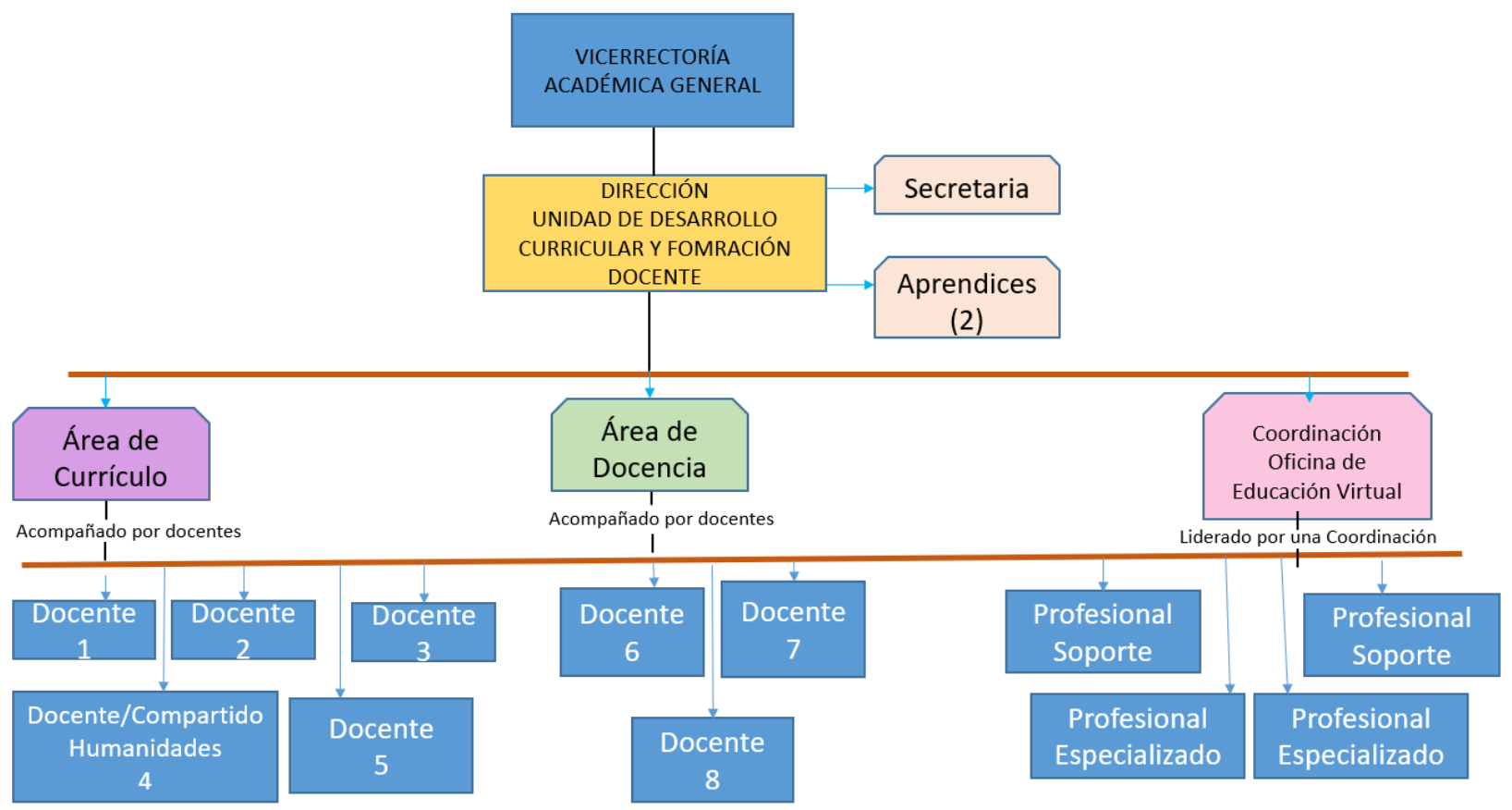

Organigrama Unidad de Desarrollo Curricular y Formación Docente, USTA.

En total las seis (6) unidades agrupan más de 60 personas de apoyo en la Sede Principal Bogotá y en las demás sedes y seccionales cuentan con más de 20 personas para un promedio de 100 profesionales y colaboradores para la gestión Multicampus.

\section{Resultados de la práctica:}

Como cambios sustantivos están el diseño de políticas, lineamientos, procesos y procedimientos para el desarrollo de las funciones universitarias, que derivan en 1) la apropiación de la comunidad docente de la propuesta formativa desde el realismo pedagógico tomista; 2) la maximización de la dedicación docente en las funciones sustantivas; 3) la implementación de un software especializado de Planeación 
Académica para la optimización de recursos docentes, físicos y tecnológicos; 4) la actualización del Estatuto Docente; 5) la definición de condiciones institucionales para fortalecer la permanencia con bienestar de los docentes; 6) la elaboración del Plan de Formación Docente 2014-2020 para el establecimiento de programas de formación permanentes y posgradual; 7) el mejoramiento de las condiciones de contratación para mantener un cuerpo docente sólido y comprometido; 8) la implementación de estrategias didácticas centradas en el aprendizaje que posibiliten la articulación de las funciones sustantivas; 9) el reconocimiento de experiencias exitosas generadas por los docentes en el marco del desarrollo de las funciones universitarias; 10) la definición de apuestas nacionales y dos campos de acción, sociedad y ambiente, que desde lo local, regional, nacional e internacional, contribuyan a la solución de problemáticas relevantes de las regiones y del país; 11) el aumento en la visibilidad e impacto de la producción académica; 12) la articulación de proyectos y estrategias de proyección social con proyectos de investigación y diseños curriculares; 13) la implementación de un Sistema Institucional de Investigación e Innovación Multicampus; 14) el diseño de la Política de Investigación; 15) la generación de estrategias para fortalecer los vínculos de las funciones sustantivas; 16) la formulación de un sistema institucional de desarrollo estudiantil; 17) la formulación de una política para los posgrados; entre otros.

El III Encuentro Académico Nacional 2019, organizado por la VAG y sus Unidades Académicas, gestó la formulación de un Modelo de Gestión Académica, desde la reflexión del cómo la Comunidad Educativa debe llegar a un consenso sobre las dinámicas de interrelación de los elementos definidos en el mismo, a partir de las políticas y lineamientos institucionales, para favorecer la definición, el manejo y la asignación de los recursos; en este sentido, debe establecer su relacionamiento con otros modelos coexistentes en la IES y con la planeación Institucional, a fin de garantizar el aseguramiento de la calidad y el desarrollo de las funciones misionales. 


\title{
IV. Modelo de Gestión Académica
}

\author{
Aproximación
}

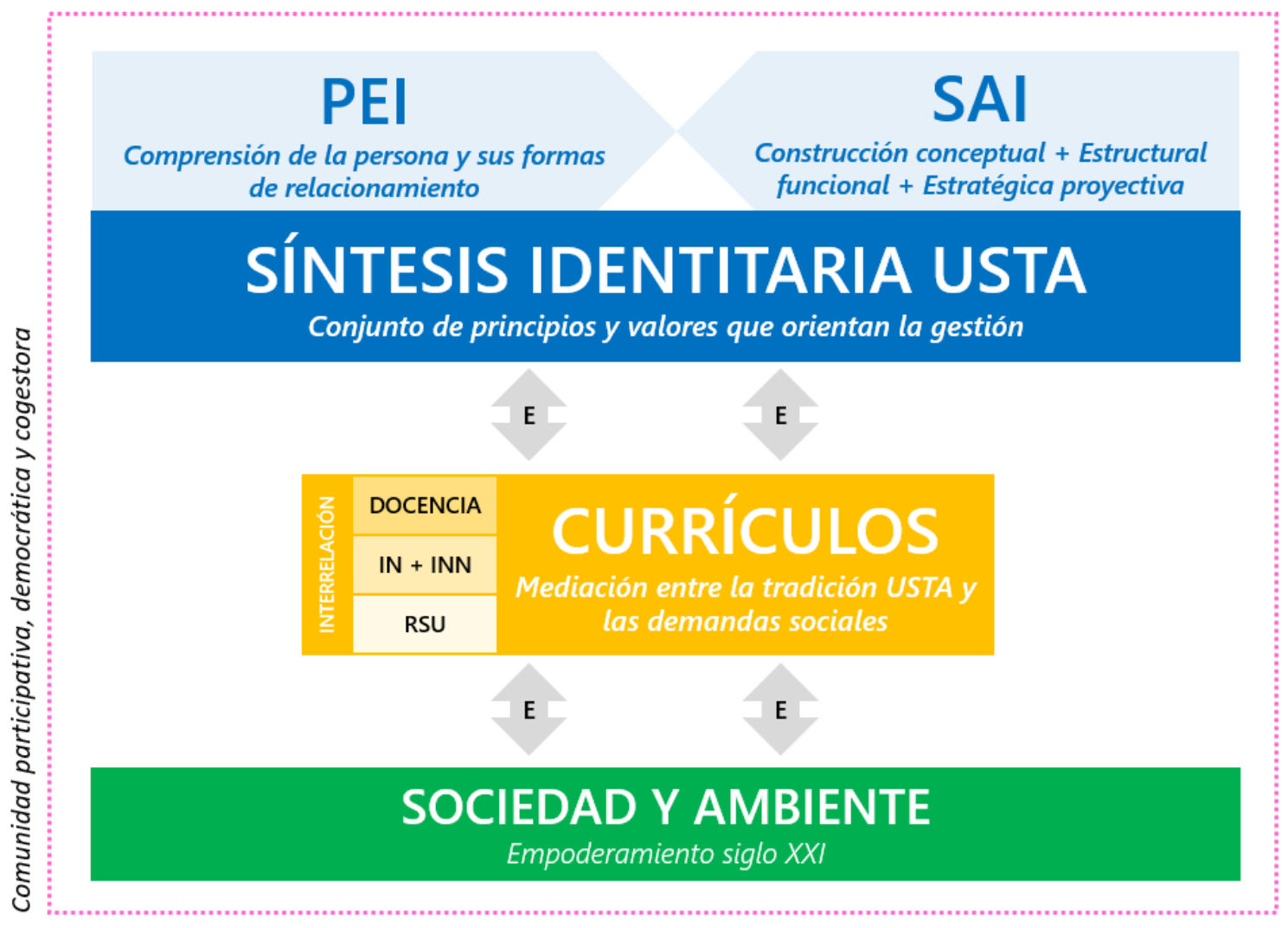

Modelo de Gestión Académica, USTA: 2019.

Adicionalmente, en el marco Multucampus tanto el Modelo Institucional de Gestión Universitaria como el Modelo de Gestión Académica, de manera articulada posibilitaron otros resultados:

- Diseño de dos rutas metodológicas para la unificación de programas académicos con igual denominación.

- Armonización académico-administrativa de 27 programas académicos de un total de 28 , con la misma denominación a través de la implementación de las rutas metodológicas fase I y II, para el logro de planes de estudio comunes y currículos integrados. 
- Formulación de un Modelo de Integración Académico-administrativa de la División de Educación Abierta y a Distancia -DUAD- con la Sede Principal Bogotá.

- Articulación de procesos académicos y proyectos conjuntos de la División de Educación Abierta y a Distancia -DUAD- con la Sede Principal Bogotá, con una apuesta importante por un Campus Virtual USTA -Colombia, que soporte el desarrollo de las funciones sustantivas: docencia, investigación y proyección social.

- Puesta en escena del Seminario Permanente en Proyecto Educativo Institucional -PEI- y Modelo Educativo Pedagógico -MEP-, en el cual a hoy docentes y decanos de todo el país se encuentran cursando la segunda cohorte.

- Implementación de un Sistema Institucional de Evaluación de los Aprendizajes -SEA-, para la modernización de los syllabus de los espacios académicos de los programas.

- Construcción de procedimientos normalizados para el apoyo a procesos académicos relacionados con la gestión curricular y el desarrollo integral docente.

- Creación del Instituto de la Paz y el Desarrollo - IPAZDE-, adscrito a la VAG, con el apoyo de diferentes facultades y unidades académicas, que pretende ser un escenario de construcción académica en torno a los procesos de reconciliación y paz en el país.

- Creación del Centro de Estudios en Pedagogía Fray José de Jesús Sedano, como una apuesta permanente de la USTA en torno a su misión formadora.

- Diseño de un Plan de Acompañamiento para fortalecer el desempeño Docente en el Aula.

- Desarrollo de proyectos de investigación de las Unidades Académicas en trabajo colegiado con diversas facultades.

- Creación del grupo de investigación en Gobernanza liderado por la VAG 
Cada una de las transformaciones que han ido sucediendo se suman a los indicadores, logros y entregables en las áreas académicas y administrativas, el número de programas ofertados, la cualificación de nuestros docentes, de los cuales hoy 174 cuentan con título de doctorado, 75 grupos de investigación reconocidos y categorizados en el Sistema Nacional de Ciencia, Tecnología e Innovación (SNCTel), 203 investigadores reconocidos por Colciencias, el aumento significativo de la producción académica con calidad e impacto, la presencia en los diferentes ránquines nacionales e internacionales, la movilidad académica de distintos actores curriculares.

Fruto de la gestión académica, a través del ejercicio de Gobernanza y el apoyo de las Unidades Académicas, la USTA ha obtenido:

- Acreditación Institucional Multicampus 2016

- Incorporación en el ranking QS STARS 2018

- Acreditación Institucional Internacional IAC - CINDA 2019

\section{Evaluación y revisión de la práctica:}

El ejercicio de evaluación de la gestión de las unidades académicas, en el marco de la gobernanza universitaria, se ha dado a través de cuatro escenarios:

\section{Establecimiento de indicadores de gestión académica desde el PIM, el PGD y el PD de cada sede y seccional}

En el momento de la formulación de cada uno de los planes que enmarcan el actuar Multicampus, se establecen indicadores de gestión que a su vez se constituyen en indicadores para la medición del quehacer de las unidades académicas. Dichos indicadores son de carácter cualitativo y cuantitativo y permiten establecer puntos de realización, así como falencias por mejorar durante la vigencia de un plan. 
La USTA en su PIM plantea seis líneas de acción:

- (1) Gobierno consolidado y fortalecido

- (2) Compromiso con el Proyecto Educativo

- (3) Proyección social e Investigación pertinentes

- (4) Enriquecimiento regional de los programas con estándares comunes

- (5) Personas que transforman sociedad

- (6) Capacidad y gestión institucional que logran la efectividad multicampus

En cada línea se plantean indicadores de medición de resultados, a manera de ejemplo, para la línea (4), un indicador es la unificación del 100\% de programas académicos con igual denominación. Esta gestión si bien es de las facultades a nivel nacional, se debe dar desde políticas y lineamientos institucionales propuestos por la VAG y la UDCFD y aprobados por los cuerpos colegiados de la USTA.

El porcentaje de cumplimiento en tres años de trabajo es del $96 \%$, lo que a su vez se constituye, a la fecha, en efectividad en el proceso y por ende en su gestión.

\section{Formulación, implementación y seguimiento a proyectos de cada unidad en sus planes de acción, con vigencia anual, y sus resultados tangibles para una USTA Multicampus}

Anualmente cada Unidad de apoyo debe presentar a la VAG, los proyectos de desarrollo de la siguiente vigencia anual. Luego de su aprobación, se inicia un seguimiento semanal a su desarrollo, lo que se constituye en ejercicio evaluativo permanente. Este a su vez busca identificar puntos de fuga, fortalezas, oportunidades de mejora, para el accionar de cada uno de los proyectos.

A manera de ejemplo, un proyecto nacional adherido a la VAG es la actualización del Estatuto Docente del 2004. El punto más álgido tuvo que ver con el escalafonamiento docente y su impacto financiero. A través del Comité de 
Unidades, que sesiona semanalmente y es presidido por la VAG, se logra establecer la evaluación de este proyecto y la manera en que se debían superar estas dos acciones dentro del proyecto. Se logra dicha actualización y ya entra en aval definitivo por el Consejo Superior, en la vigencia 2019-2.

3. Heteroevaluación de las directivas de la USTA a la gestión de los diferentes equipos de trabajo de las seis unidades de apoyo académico

El Estatuto Orgánico (2018) faculta al Consejo Superior a evaluar la gestión de las unidades de apoyo académico, previo análisis de la VAG. Dicho análisis ha posibilitado que las unidades sean re-pensadas y re-dimensionadas permanentemente, y siempre en búsqueda del mejoramiento continuo, de cara a las necesidades institucionales, locales, nacionales e internacionales de la educación superior. Esta evaluación se realiza de manera anual.

\section{Metaevaluación de la evaluación de unidades y la ejecución de su plan} de acción, lo que posibilita redimensionar la estructura y funcionamiento de cada unidad

Compete a la VAG, evaluar la manera de evaluar las unidades académicas. Dicha evaluación hace referencia a considerar la evaluación como un ejercicio de aprendizaje y de aseguramiento de la calidad. En este sentido, se toman como insumos la evaluación 360 que se hace a directores de las unidades y la percepción de los diferentes actores institucionales frente al rendimiento y acompañamiento de las unidades a la academia. De esta forma y de manera anual, se revisa este proceso evaluativo que permite mejoras tanto en el relacionamiento de las unidades con facultades y departamentos académicos así como con la alta dirección institucional. He aquí un proceso de autorregulación permanente, fruto de la autoevaluación y demás ejercicios evaluativos. 


\section{Carácter Innovador de la práctica:}

Su carácter innovador de la práctica radica en tres asuntos:

1. Por una parte, las unidades de gestión académica son órganos consultores y asesores de la VAG, lo que permite que a nivel jerárquico gocen de un estatus de apoyo más que de órgano de dirección y control. Esto hace que el aprendizaje por pares, los consensos, los ejercicios participativos y democráticos, permitan gestionar a nivel nacional una universidad multicampus.

2. Por otra, al contar con unidades homólogas en todo el país, en sedes y seccionales de la USTA, se genera una acción multicampus sintonizada, democrática y participativa. Cada homólogo de una unidad se constituye en miembro de mesas nacionales, que sesionan presididas por la VAG, para una gestión nacional articulada y sinérgica. Se trata entonces de vincular a través de mesas nacionales toda la gestión universitaria. Se cuenta a la fecha con mesas nacionales de Investigación, de currículo y docencia, de Proyección Social, entre otras.

3. La gestión de las unidades ha posibilitado que las funciones universitarias se manejen de manera articulada, no aislada por dependencias o instancias, sino por procesos enmarcados en planes y proyectos visionarios, y de largo alcance. Esto a su vez ha generado sistematización de experiencias y buenas prácticas, que a su vez han sido presentadas en diferentes escenarios nacionales e internacionales. Las mismas se constituyen en espacios de aprendizaje y cualificación que fomentan no solo la institucionalidad sino la excelencia de los diversos procesos de gestión.

4. La gestión de las Unidades y su carácter bidireccional favorece que los niveles estratégico y operativo se realimenten en doble vía. 


\section{Divulgación de la práctica:}

La divulgación tiene dos vertientes:

1. A nivel interno Institucional: a través de los diversos canales de comunicación, entre otros: página Web, Mesas Nacionales, Comités Particulares de las funciones sustantivas, Sistema Institucional de Aseguramiento de la Calidad -SIAC-, foros, claustros docentes y administrativos, jornadas de cualificación, seminarios y congresos Institucionales, documentos institucionales divulgados con los diferentes actores académicos en jornadas de acompañamiento (ejemplo: Lineamientos para el Diseño y la Actualización Curricular, 2015; Política de Investigación, 2019). De igual forma se han elaborado artículos publicados en revistas indexadas y de divulgación, así como libros y capítulos de libro. A manera de ejemplo se encuentra el libro: Gestión curricular y desarrollo de competencias en estudiantes y docentes apuesta por la calidad universitaria, 2019: ediciones USTA.

2. A nivel externo nacional e internacional: participación en eventos académicos nacionales e internacionales, entre otros con la presentación de buenas prácticas de gestión académico-administrativa: Universidad del Norte (Barranquilla); Universidad Nacional de Colombia; Congreso Internacional de Psicología y Educación 2018, XXVIII Simposio Internacional de Estadística, 2018, Simposio de Ciencias de la Educación de la $9^{\circ}$ Conferencia Científica Internacional de la Universidad de Holguín, 2019; otros congresos internacionales en y para Educación Superior en Perú, Argentina, Cuba y México; misiones académicas a Europa, así como la escritura de artículos publicados en revistas indexadas y de divulgación. 
A manera de ejemplo:

\begin{tabular}{|c|c|c|}
\hline Nombre del producto & $\begin{array}{l}\text { Evento en el cual se } \\
\text { presenta la ponencia }\end{array}$ & $\begin{array}{l}\text { Lugar en el cual } \\
\text { se presenta }\end{array}$ \\
\hline $\begin{array}{c}\text { Unidad de Desarrollo } \\
\text { Curricular y Formación } \\
\text { Docente: Una experiencia de } \\
\text { Gestión Académica de la } \\
\text { Universidad Santo Tomás para } \\
\text { la alta calidad Multicampus y la } \\
\text { institucionalidad" }\end{array}$ & $\begin{array}{c}\text { Primer Encuentro } \\
\text { Latinoamericana de } \\
\text { Enseñanza- aprendizaje en } \\
\text { Educación Superior. } \\
\text { CEDU- Universidad del Norte } \\
2017\end{array}$ & Barranquilla \\
\hline $\begin{array}{l}\text { Gestión del Currículo en la } \\
\text { Educación Superior: } \\
\text { Experiencia de la USTA en el } \\
\text { marco de la acreditación } \\
\text { Multicampus. }\end{array}$ & $\begin{array}{c}\text { Primer Encuentro } \\
\text { Latinoamericana de } \\
\text { Enseñanza- aprendizaje en } \\
\text { Educación Superior.CEDU- } \\
\text { Universidad del Norte } 2017\end{array}$ & Barranquilla \\
\hline $\begin{array}{l}\text { Gestión Curricular: desde los } \\
\text { estudios de pertinencia hasta } \\
\text { la implementación del } \\
\text { microcurrículo. } \\
\text { Una apuesta USTA por la } \\
\text { responsabilidad social en el } \\
\text { marco de la formación integral }\end{array}$ & $\begin{array}{c}\text { Congreso de Psicología y } \\
\text { Responsabilidad Social, } 2017\end{array}$ & Bogotá \\
\hline $\begin{array}{c}\text { Gestión del currículo en } \\
\text { Educación Superior: Desde la } \\
\text { política Institucional hasta el } \\
\text { diseño de microcurriculos por } \\
\text { competencias y dimensiones } \\
\text { de la acción humana }\end{array}$ & $\begin{array}{c}\text { XIII congreso Internacional } \\
\text { sobre el Enfoque Basado en } \\
\text { Competencias } \\
2017\end{array}$ & Cartagena \\
\hline $\begin{array}{l}\text { Campos de acción de la } \\
\text { Universidad Santo Tomás - } \\
\text { USTA- Colombia: una apuesta } \\
\text { por la formación integral desde } \\
\text { la articulación de las funciones } \\
\text { sustantivas en el maraco de la } \\
\text { diversidad regional del país }\end{array}$ & $\begin{array}{l}\text { VIII Encuentro Nacional y V } \\
\text { Latinoamericano La } \\
\text { Universidad como objeto de } \\
\text { investigación "La Reforma } \\
\text { Universitaria entre dos siglos" } \\
2017\end{array}$ & Argentina \\
\hline
\end{tabular}




\begin{tabular}{|c|c|c|}
$\begin{array}{c}\text { Prácticas de excelencia } \\
\text { docente: una construcción } \\
\text { innovadora para la Universidad } \\
\text { Santo Tomás -USTA- En } \\
\text { clave de transformación social }\end{array}$ & $\begin{array}{c}\text { VIII Encuentro Nacional y V } \\
\text { Latinoamericano La } \\
\text { Universidad como objeto de } \\
\text { investigación "La Reforma } \\
\text { Universitaria entre dos siglos" }\end{array}$ & Argentina \\
\hline $\begin{array}{c}\text { Gestión Curricular en } \\
\text { Universidades multicampus: } \\
\text { Unidad en la diversidad } \\
\text { regional, en el marco de la } \\
\text { flexibilidad }\end{array}$ & $\begin{array}{c}\text { I Seminario Internacional de } \\
\text { Currículo y Educación Superior }\end{array}$ & Medellín \\
\hline $\begin{array}{l}\text { Pedagogía Problémica: un } \\
\text { enfoque desde el Modelo } \\
\text { Educativo Pedagógico de la } \\
\text { Universidad Santo Tomás y su } \\
\text { articulación docencia, } \\
\text { investigación y proyección } \\
\text { social en y para la calidad de } \\
\text { la educación superior }\end{array}$ & $\begin{array}{c}\text { V Congreso Internacional de } \\
\text { Psicología y Educación }\end{array}$ & Bogotá \\
\hline
\end{tabular}

